\title{
On the Phase Structure of the Compact Abelian Lattice Higgs Model
}

\author{
João C. A. Barata \\ Instituto de Física da Universidade de São Paulo, Caixa Postal 20516, \\ Cep. 01498. São Paulo, SP. Brasil
}

\begin{abstract}
This paper studies the vacuum overlap order parameter proposed by Fredenhagen and Marcu in the case of the compact $U(1)$ gauge model with the Wilson action coupled to a Higgs field with fixed length $|\phi|=1$. The existence of two distinct phases in $D$ space-time dimensions $(D \geq 4)$ is established.
\end{abstract}

\section{Introduction}

Gauge theories on the lattice are an important branch of research of Quantum Field Theory. They offer many advantages for theoretical and numerical studies, especially since they provide one of the few known consistent non-strictly perturbative methods of regularization of gauge theories. Their most important quality reveals in the analysis and understanding of non-perturbative phenomena, like the Higgs mechanism, the problem of confinement and that of triviality of some four dimensional models involving scalar (Higgs) fields. They have also been used as a starting point to the construction of gauge models on a continuous space-time.

The question we treat here is related to that of the existence of charged states in lattice gauge theories, in particular in models with scalar fields coupled to gauge fields.

In a study of the $\mathbb{Z}(2)$-Higgs model [1] Fredenhagen and Marcu were able to construct in the Coulomb region of its phase diagram, for the first time, charged sectors of the associated quantum system (see also [12]). As a consequence of their analysis, these authors proposed a non-local order parameter to distinguish phases in lattice gauge theories coupled to matter fields. This order parameter, frequently named after his authors or donoted "Voop" (for "vacuum ovelap order parameter"), essentially measures the limit value of projections on the vacuum of a suitably constructed sequence of normalized dipole states with bounded energy. Its particular importance, in contrast to other order parameters used in lattice gauge theories, resides in its direct physical interpretation and particularly in its 
sensitivity for models involving matter fields. We will not enter into details about its motivation here, preferring to refer the interested reader to the references $[3,1$, $2,4]$. This order parameter has also been object of intensive numerical analysis by many groups and showed to be quite useful for the study of the phase structure of gauge models through numerical simulations (see f.i. [16] and other references therein).

Using this order parameter we will show the existence of two distinguished phases in the phase diagram of the compact $U(1)$ model with Higgs fields with a Wilson action in at least four space-time dimensions, which we interpret as a Higgs-confinement phase and a Coulomb phase respectively. This result confirms the expected picture (see [6]). We note that the existence of the Coulomb phase has already been established by Kondo in [5]. Our method is strongly based on [1], where this order parameter was analysed in the case of the $\mathbb{Z}(2)$ gauge Higgs model.

We have to mention here the many analytical studies performed in the non-compact version of this model which also provided an almost complete understanding of the structure of its phase diagram (see [7] for a review).

\subsection{Description of the Model}

We describe now briefly the model we will consider (see also [15]). We fix a $d+1$-dimensional $(d \geq 1)$ lattice $L=\mathbb{Z}^{d+1}$. We call $L^{1}$ the set of all positively oriented bonds on $L$ and $L^{2}$ the set of all positively oriented plaquettes (the same notation will be extended to sub-sets of $L$ ). We represent the points of $L$ by coordinates $\left(x^{0}, \underline{x}\right), x \in L^{1}$, the zero direction being the euclidean time direction. We attach to each bond $b \in L$ a gauge field $U(b)=e^{i \theta(b)}$ with $-\pi<\theta(b) \leq \pi$, and to each $x \in L$ a scalar field with fixed length $\phi(x)=e^{i \tau(x)}$, with $-\pi<\tau(x) \leq \pi$.

For $\Lambda \subset L,|\Lambda|>\infty$ we define the action $S_{\Lambda}$ as

$$
S_{\Lambda}:=-\beta_{g} \sum_{p \in \Lambda^{2}}[\cos (\theta(p))+k]-\beta_{h} \sum_{p \in \Lambda^{1}}[\cos (-\partial \tau(b)+\theta(b))+c]
$$

with $\partial \tau(b)=\tau\left(x_{2, b}\right)-\tau\left(x_{1, b}\right)$, where $x_{2, b}, x_{1, b}$ are the two extremal points of $b$ with $\left(x_{2, b}\right)^{b}>\left(x_{1, b}\right)^{b}$ where $\left(x_{i, b}\right)^{b}$ is the component of $x_{i, b}, i=1,2$ in the direction defined by the positive sense of $b$, where

$$
\theta(p)=\sum_{b \in p}(p \mid b) \theta(b),
$$

where $(p \mid b)= \pm 1$ is the relative orientation of $b$ in $p$ and where and $k$ and $c$ are constants. The coupling constants $\beta_{g}$ and $\beta_{h}$ are real and positive.

At finite volume, the expectation value of local gauge invariant observables is defined by

$$
\langle A\rangle_{\Lambda}:=Z_{\Lambda}^{-1} \int_{-\pi}^{\pi}[d \tau]_{\Lambda}[d \theta]_{\Lambda^{1}} A(\tau, \theta) e^{-S_{\Lambda}},
$$

where $[d \tau]_{\Lambda}=\prod_{x \in \Lambda} d \tau(x)$ and $[d \theta]_{\Lambda^{1}}=\prod_{b \in \Lambda^{1}} d \theta(b)$, with $\langle 1\rangle_{\Lambda}=1$. 
We pass to the so-called unitary gauge by defining

$$
u(b)=\theta(b)-\partial \tau(b),
$$

and the expectation value becomes

$$
\langle A\rangle_{\Lambda}:=Z_{\Lambda}^{-1} \int_{-\pi}^{\pi}[d u]_{\Lambda^{1}} A(u) e^{-S_{\Lambda}},
$$

with

$$
S_{\Lambda}:=-\beta_{g} \sum_{p \in \Lambda^{2}}[\cos (u(p))+k]-\beta_{h} \sum_{p \in \Lambda^{1}}[\cos (u(b))+c] .
$$

We will extend the notation above and denote, for any finite $B \subset L^{1}$, $[d u]_{B}=\prod_{b \in B} d u(b)$.

For each fixed observable $A$ the thermodynamical limit is defined by

$$
\langle A\rangle:=\lim _{\Lambda \uparrow L}\langle A\rangle_{\Lambda},
$$

under suitable sequences and defines a translation invariant state in the algebra of observables which we call the vacuum state.

We call $T_{(a, b)}^{(c, b)}$ the set of bounds of $L^{1}$ contained in the line segment joining the points $(c, \underline{b})$ and $(a, \underline{b})$.

For all $r \in \mathbb{N}, r \geq 1$ we define the sets

$$
M_{r}:=\left\{b \in L^{1}: b \in T_{-(r, \underline{0})}^{(r, \underline{0})} \cup T_{(r, \underline{0})}^{\left(r, 2 \hat{x}^{1}\right)} \cup T_{(r, \underline{0})}^{\left(r, 2 r \hat{x}^{1}\right)} \cup T_{\left(r, 2 r \hat{x}^{1}\right)}^{\left(-r, 2 \hat{x}^{1}\right)} \cup T_{\left(-r, 2 r \hat{x}^{1}\right)}^{(-r, 0)}\right\}
$$

and

$$
N_{r}:=\left\{b \in L^{1}: b \in T_{(0, \underline{0})}^{(r, \underline{0})} \cup T_{(r, \underline{0})}^{\left(r, 2 r \hat{x}^{1}\right)} \cup T_{\left(r, 2 r \hat{x}^{1}\right)}^{\left(0,2 \hat{x}^{1}\right)}\right\}
$$

where $\hat{x}^{1}$ is the unit vector $(0,1,0, \ldots, 0)$. we define also $\vartheta N_{r}$ which is obtained by reflecting $N_{r}$ on the hyperplane $x^{0}=0$. See Fig. 1 .

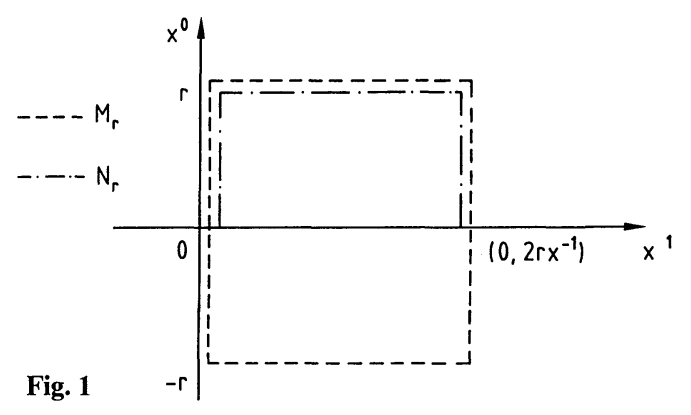

The sets $M_{r}, N_{r}$, and $\vartheta N_{r}$ will be considered to be positively (clockwise) oriented. We define

$$
u_{M_{r}}:=\sum_{b \in M_{r}}\left(M_{r} \mid b\right) u(b)
$$


and

$$
u_{N_{r}}:=\sum_{b \in N_{r}}\left(N_{r} \mid b\right) u(b)
$$

$\left(M_{r} \mid b\right)= \pm 1$ being the relative orientation of $b$ in $M_{r}$, etc.

We will then consider the order parameter $\varrho_{\text {voop }}$ given by

where

$$
\varrho_{\text {voop }}:=\lim _{r \rightarrow \infty} \varrho_{\text {voop }}^{(r)} \text {, }
$$

$$
\varrho_{\text {voop }}^{(r)}:=\frac{\left\langle e^{i u_{N_{r}}}\right\rangle^{2}}{\left\langle e^{i u_{M_{r}}}\right\rangle} .
$$

Our interest is to study the behavior of $\varrho_{\text {voop }}$ in different regions of the $\left(\beta_{g}, \beta_{h}\right)$ phase diagram, whose expected phase structure in four or more space-time dimensions is described in Fig. 2. The region $I$ is the Higgs-confinement phase and the region $I I$ is the Coulomb phase. Our results were established on regions $I_{c}$ and $I I_{c}$.

Fig. 2

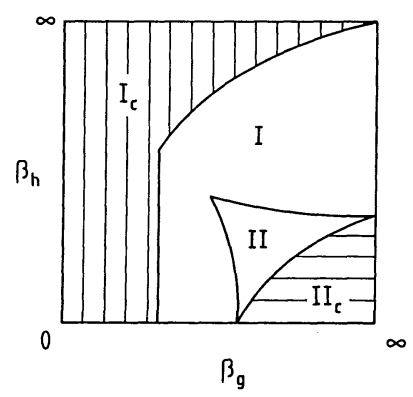

\subsection{The Coulomb Phase}

First we show the existence of a phase where $\varrho_{\text {voop }}$ is identically zero. The existence of this phase was first established in [5]. We present a new proof of this fact $^{1}$.

According to well known correlation inequalities (see $[10,13]$ ), which unfortunately do not hold for non-abelian Higgs models, we have for the hole phase diagram

and

$$
\left\langle e^{i u_{N_{r}}}\right\rangle\left(\beta_{g}, \beta_{h}\right) \leq\left\langle e^{i u_{N_{r}}}\right\rangle\left(\infty, \beta_{h}\right)
$$

$$
\left\langle e^{i u_{M_{r}}}\right\rangle\left(\beta_{g}, \beta_{h}\right) \leq\left\langle e^{i u_{M_{r}}}\right\rangle\left(\beta_{g}, 0\right) .
$$

But $\left\langle e^{i u_{N_{r}}}\right\rangle\left(\infty, \beta_{h}\right)$ is equal to the two point function $\left\langle\phi(0) \phi\left(2 r \hat{x}^{1}\right)\right\rangle^{X Y}\left(\beta_{h}\right)$ of the $X Y$-model which, for $\beta_{h}$ small enough, has the bound

$$
\left\langle\phi(0) \phi\left(2 r \hat{x}^{1}\right)\right\rangle^{X Y}\left(\beta_{h}\right) \leq c_{1} e^{-m\left(\beta_{h}\right) 2 r},
$$

\footnotetext{
$\overline{1 \text { The argument }}$ is already contained in [1]. I am indebted to K. Fredenhagen who presented it to me in this form
} 
where $c_{1}$ is a constant and $m\left(\beta_{h}\right)>0$ is the mass-gap of the model and is, for $\beta_{h}$ small, of the form $m\left(\beta_{h}\right)=-c_{2} \ln \left(\beta_{h} / \beta_{0}\right)+f\left(\beta_{h}\right)$ for constants $c_{2}$ and $\beta_{0}$, where $f\left(\beta_{h}\right)$ is analytical.

Beyond this for $d+1 \geq 4$ we have for $\beta_{g}$ large enough $[8,9]$

$$
\left\langle e^{i u_{M_{r}}}\right\rangle\left(\beta_{g}, 0\right) \geq c_{3} e^{-\alpha\left(\beta_{g}\right) r} .
$$

So, for $d+1 \geq 4, \beta_{g}$ large enough and $\beta_{h}$ small enough we get

$$
\varrho_{\mathrm{voop}}^{(r)} \leq\left(c_{1}^{2} / c_{2}\right) e^{-\left(2 m\left(\beta_{h}\right)-\alpha\left(\beta_{g}\right)\right) r},
$$

and since for $\beta_{h}$ small $2 m\left(\beta_{h}\right)>\alpha\left(\beta_{g}\right)$ we get $\varrho_{\text {voop }}=0$ for a region as region $I I_{c}$ in Fig. 2. Actually [8] also established that $\lim _{\beta_{g} \rightarrow \infty} \alpha\left(\beta_{g}\right)=0$, and so region $I I_{c}$ extends up to the critical point on the line $\beta_{g}=\infty$.

In order to study $\varrho_{\text {voop }}^{(r)}$ in the confinement-Higgs phase we will construct a polymer expansion for $\left\langle e^{i u_{M_{r}}}\right\rangle$ and $\left\langle e^{i u_{N_{r}}}\right\rangle$ and a cluster expansion for $\varrho_{\text {voop }}^{(r)}$ and study its region of convergence in the $\left(\beta_{g}, \beta_{h}\right)$-phase diagram.

\section{The Polymer Expansion}

Let $A$ be one of the sets $M_{r}, N_{r}$ or $\vartheta N_{r}$, for some fixed $r \in \mathbb{N}$. In order to motivate this expansion we note that for $\beta_{g}=0$ one has simply

$$
\left\langle e^{i u_{A}}\right\rangle_{\Lambda}=C_{1}\left(\beta_{h}\right)^{|A|}
$$

with

$$
C_{n}\left(\beta_{h}\right):=I_{n}\left(\beta_{h}\right) / I_{0}\left(\beta_{h}\right),
$$

where $I_{n}(x):=(2 \pi)^{-1} \int_{-\pi}^{\pi} e^{x \cos \theta} \cos (n \theta) d \theta, n \in \mathbb{N}$, are the modified Bessel functions, and so $\varrho_{\text {voop }}=1$ for $\beta_{g}=0, \beta_{h} \neq 0$.

We have for $\Lambda^{1} \supset A$,

$$
\left\langle e^{i u_{A}}\right\rangle_{\Lambda}=Z_{A}^{-1} \sum_{\mathscr{P} \subset \Lambda^{2}} \int_{-\pi}^{\pi}[d u]_{\Lambda^{1}}\left\{\prod_{b \in \Lambda^{1}} e^{\beta_{h} \cos u(b)+c}\right\} e^{i u_{A}} \prod_{p \in \mathscr{P}} \varrho_{p}(u(p)),
$$

with

$$
\varrho_{p}(u(p)):=e^{\beta_{g} \cos u(p)+k}-1 .
$$

We call $\partial \mathscr{P}$ the set of all bonds of $\mathscr{P} \subset \Lambda^{2}$. We choose $c$ so that $\int_{-\pi}^{\pi} d u e^{\beta_{h} \cos u+c}=1$. We get

$$
\begin{aligned}
\left\langle e^{i u_{A}}\right\rangle_{\Lambda}= & Z_{\Lambda}^{-1} \sum_{\mathscr{P} \subset \Lambda^{2}} \int_{-\pi}^{\pi}[d u]_{\Lambda^{1} \backslash \partial \mathscr{P}}\left\{\prod_{b \in \Lambda^{1} \backslash \partial \mathscr{P}} e^{\beta_{h} \cos u(b)+c}\right\} e^{i u_{A \backslash \partial \mathscr{P}}} \\
& \times \int_{-\pi}^{\pi}[d u]_{\partial \mathscr{P}}\left\{\prod_{b^{\prime} \in \partial \mathscr{P}} e^{\beta_{h} \cos u\left(b^{\prime}\right)+c}\right\} e^{i u_{A \cap \partial \mathscr{P}}} \prod_{p \in \mathscr{P}} \varrho_{p}(u(p)),
\end{aligned}
$$


which may be written as

$$
\left[C_{1}\left(\beta_{h}\right)\right]^{|A|} Z_{\Lambda}^{-1} \sum_{\mathscr{P} \subset \Lambda^{2}} \int_{-\pi}^{\pi}[d u]_{\partial \mathscr{P}}\left\{\prod_{b \in \partial \mathscr{P}} \frac{e^{\beta_{h} \cos u(b)}}{2 \pi I_{0}\left(\beta_{h}\right)}\right\}\left[\prod_{b^{\prime} \in A \cap \partial \mathscr{P}} \frac{e^{i\left(A \mid b^{\prime}\right) u\left(b^{\prime}\right)}}{C_{1}\left(\beta_{h}\right)}\right] \prod_{P \in \mathscr{P}} \varrho_{p}(u(p)) .
$$

Now we define connectivity relations for plaquettes in $\Lambda^{2}$. Two plaquettes are said to be connected if they have at least one common bond. So $\mathscr{P} \subset \Lambda^{2}$ may be decomposed into a sum of connected sets of plaquettes called polymers: $\mathscr{P}=\sum_{i \geq 1} \gamma_{i}$, where $\sum$ denotes disjoint union.

We can then factorise (24) in terms of activities associated to each polymer $\gamma$ :

$$
\left\langle e^{i u_{A}}\right\rangle_{\Lambda}=\left[C_{1}\left(\beta_{h}\right)\right]^{|A|} \frac{\sum_{\Gamma \in G_{a d}^{A}} \prod_{\gamma \in \Gamma} \mu_{A}(\gamma)}{\sum_{\Gamma \in G_{a d}^{A}} \prod_{\gamma \in \Gamma} \mu_{\emptyset}(\gamma)}
$$

where $G_{a d}^{\Lambda}$ is the set of all sets of compatible polymers contained in $\Lambda$ (see Appendix) and

$$
\mu_{A}(\gamma):=\int_{-\pi}^{\pi}[d u]_{\partial \gamma}\left\{\prod_{b \in \partial \gamma} \frac{e^{\beta_{h} \cos u(b)}}{2 \pi I_{0}\left(\beta_{h}\right)}\right\}\left[\prod_{c \in A \cap \partial \gamma} \frac{e^{i(A \mid c) u(c)}}{C_{1}\left(\beta_{h}\right)}\right] \prod_{p \in \gamma} \varrho_{p}(u(p))
$$

for $\gamma \neq \emptyset$, with $\mu_{A}(\emptyset)=1$. Above $\partial \gamma$ is the set of all bonds belonging to plaquettes in $\gamma$.

As we describe in Sect. 3 our interest is to develop a cluster expansion for (25). We need, as described in the Appendix, an upper bound for

$$
\left\|\mu_{c}\right\|:=\max _{A \in\left\{M_{r}, N_{r}, \emptyset\right\}} \sup _{\gamma \neq \emptyset}\left|\mu_{A}(\gamma)\right|^{1 /|\gamma|},
$$

where $|\gamma|$ is the cardinality of $\gamma$, i.e., in this case the number of plaquettes in $\gamma$.

We develop now a Fourier expansion of the factors $e^{\beta_{h} \cos u(b)} / 2 \pi I_{0}\left(\beta_{h}\right)$ and $\varrho_{p}(u(p))$ found in (26). We write

$$
\varrho_{p}(u(p))=\sum_{m_{p} \in \mathbb{Z}} d_{m_{p}}\left(\beta_{g}\right) e^{i m_{p} u(p)} / 2 \pi
$$

with $d_{m}\left(\beta_{g}\right):=\int_{-\pi}^{\pi} d u\left(e^{\beta_{g} \cos u+k}-1\right) e^{i m u}$ and

$$
e^{\beta_{h} \cos u(b)} / 2 \pi I_{0}\left(\beta_{h}\right)=\sum_{n_{b} \in \mathbb{Z}} C_{n_{b}}\left(\beta_{h}\right) e^{i n_{b} u(b)} / 2 \pi
$$

with $C_{n}\left(\beta_{h}\right)$ as in (20).

Note that $C_{n}(x)=C_{-n}(x), C_{0}(x)=1$ and $C_{n} \leq 1 \forall n \in \mathbb{Z}$. Beyond this $\lim _{x \rightarrow \infty} C_{n}(x)=1$ and for $n \neq 0, C_{n}(0)=0$.

Defining

$$
k(a, b):= \begin{cases}(A \mid b) & \text { if } b \in A \\ 0 & \text { otherwise }\end{cases}
$$


we get, using (28) and (29),

$$
\mu_{A}(\gamma)=C_{1}\left(\beta_{h}\right)^{-|A| \mathrm{\cap} \partial \gamma \mid} \sum_{\left\{m_{p}\right\}_{p \in \gamma}} \prod_{p \in \gamma} \frac{d_{m_{p}}\left(\beta_{g}\right)}{2 \pi} \prod_{b \in \partial \gamma} C_{\left(\partial_{\gamma}^{*} m\right)_{b}+k(A, b)}\left(\beta_{h}\right),
$$

where

$$
\left(\partial_{\gamma}^{*} M\right)_{b}:= \begin{cases}\sum_{p \ni b, p \in \gamma}(p \mid b) m_{p} & \text { if } b \in \partial \gamma \\ 0 & \text { otherwise }\end{cases}
$$

To simplify the notation we define

$$
j(A, \gamma, b):=\left(\partial_{\gamma}^{*} m\right)_{b}+k(A, b) .
$$

In order to get a good control of the convergence region of the corresponding cluster expansion (see below) for arbitrarily small, but strictly positive values of $\beta_{h}$ we have to be especially careful (in the case $A \neq \emptyset$ ) with the factor $C_{1}\left(\beta_{h}\right)^{-|A \cap \partial \gamma|}$ occurring in (31). This factor could damage the desired bound (71) for (27). The strategy to follow is to compensate this factor at least partially by suitably chosen factors $C_{j(A, \gamma, b)}\left(\beta_{h}\right)$ occurring in each term in the sum in (31).

\subsection{The Region of Convergence}

First we needed some definitions. For $r$ fixed we define the sequences of oriented hyperplanes $\left\{S_{a}\right\}_{a=-r}^{r-1}$ and $\left\{R_{b}\right\}_{b=0}^{2 r-1}$ by

$$
\begin{aligned}
& S_{a}:=\left\{x \in \mathbb{R}^{d+1}, x^{0}=a+1 / 2\right\}, \\
& R_{b}:=\left\{x \in \mathbb{R}^{d+1}, x^{1}=b+1 / 2\right\} .
\end{aligned}
$$

We denote by $\partial^{*} S_{a}$ (respectively $\partial^{*} R_{b}$ ) the set of all bonds of $L^{1}$ which are intercepted by $S_{a}\left(R_{b}\right)$ and call, for $b \in \partial^{*} S_{a},\left(S_{a} \mid b\right)$ the orientation of $b$ relative to $S$ [correspondingly for $b^{\prime} \in \partial^{*} R_{b}$ we define $\left(R_{b} \mid b^{\prime}\right)$ ].

For a given polymer $\gamma$ and for given $S_{a}$ (respectively $R_{b}$ ) as above we consider the set $\partial \gamma \cap \partial^{*} S_{a}$ (respectively $\partial \gamma \cap \partial^{*} R_{b}$ ). Two bonds $b_{1}, b_{2} \in \partial \gamma \cap \partial^{*} S_{a}$ (respectively $\in \partial \gamma \cap \partial^{*} R_{b}$ ) are called connected if there exists $p \in \gamma$ such that $b_{1} \in \partial p$ and $b_{2} \in \partial p$. Call $\left\{\left(S_{a}, \gamma ; i\right)\right\}_{i=1}^{n}$ (respectively $\left.\left\{\left(R_{b} \gamma ; j\right)\right\}_{j=1}^{m}\right)$ the set of all connected components of $\partial \gamma \cap \partial^{*} S_{a}\left(\partial \gamma \cap \partial^{*} R_{b}\right)$ by the connectivity relation above. Of course $\partial \gamma \cap \partial^{*} S_{a}=\sum_{i=1}^{n}\left(S_{a}, \gamma ; i\right)\left(\partial \gamma \cap \partial^{*} R_{b}=\sum_{j=1}^{m}\left(R_{b}, \gamma ; j\right)\right)$.

Define

$$
\begin{aligned}
& V_{r}^{a}:=\left\{\partial^{*} S_{a} \cap V_{r} \cap A\right\}, \\
& H_{r}^{b}:=\left\{\partial^{*} R_{b} \cap H_{r} \cap A\right\},
\end{aligned}
$$

with

$$
\begin{gathered}
V_{r}:=T_{(-r, \underline{0})}^{(r, \underline{0})} \cup T_{\left(r, 2 r \hat{x}^{1}\right)}^{\left(-r, 2 r \hat{x}^{1}\right)}, \\
H_{r}:=T_{(r, \underline{0})}^{\left(r, 2 r \hat{x}^{1}\right)} \cup T_{\left(-r, 2 r \hat{x}^{1}\right)}^{(-r, \underline{0})} .
\end{gathered}
$$


If for a given $a$ (respectively $b),\left|V_{r}^{a}\right|=2$ (respectively $\left|H_{r}^{b}\right|=2$ ) we call the two elements of $V_{r}^{a}$ (respectively of $H_{r}^{b}$ ) $\gamma$-associated if both belong to the same connected component $\left(S_{a}, \gamma ; i\right)$ for some $i$ [respectively $\left(R_{b}, \gamma ; j\right)$ for some $j$ ].

We will denote by $T_{r}$ the set of all $\gamma$-associated bonds of the set $A$ and define $C_{V}:=T_{r} \cap V_{r}, C_{H}:=T_{r} \cap H_{r}$.

We also define

$$
\begin{aligned}
& D_{V}:=\left[(\partial \gamma \cap A) \backslash T_{r}\right] \cap V_{r}, \\
& D_{H}:=\left[(\partial \gamma \cap A) \backslash T_{r}\right] \cap H_{r},
\end{aligned}
$$

In words $C_{V}\left(C_{H}\right)$ is the set of $\gamma$-associated vertical (horizontal) bonds and $D_{V}$ $\left(D_{H}\right)$ are the vertical (horizontal) elements on which are not $\gamma$-associated to another element of $\partial \gamma \cap A$.

Clearly $\partial \gamma \cap A=C_{V}+C_{H}+D_{V}+D_{H}$, where + denotes disjoint union.

To find a majorization for $\left|\mu_{A}(\gamma)\right|^{1 /|\gamma|}$ we will make use of the following three lemmas.

Lemma 2.1. For any fixed polymer $\gamma$ and for $C_{V}$ and $C_{H}$ as defined above (depending on $\gamma$ ) we have

$$
|\gamma| \geq\left|C_{V}+C_{H}\right| \frac{r}{2}
$$

Proof. If $\left|C_{V}+C_{H}\right|=0$ the relation is trivial. If not we argue as follows. First note that $\left|C_{H}\right|,\left|C_{V}\right| \leq 4 r$. By pure geometrical reasoning we find the bound

$$
|\gamma| \geq \frac{\left|C_{H}\right|}{2}(2 r)+\frac{\left|C_{V}\right|}{2}(2 r)-\frac{\left|C_{V}\right|\left|C_{H}\right|}{4}
$$

If $\left|C_{V}\right|$ or $\left|C_{H}\right|=0$ the relation (42) follows immediately. Otherwise (43) says that

$$
\begin{aligned}
|\gamma| & \geq r\left|C_{H}+C_{V}\right|-\frac{1}{4}\left|C_{H}+C_{V}\right|\left(\frac{\left|C_{H}\right|\left|C_{V}\right|}{\left|C_{H}\right|+C_{V} \mid}\right) \\
& =r\left|C_{H}+C_{V}\right|-\frac{1}{4}\left|C_{H}+C_{V}\right|\left(\frac{1}{\left|C_{H}\right|^{-1}+\left|C_{V}\right|^{-1}}\right) \\
& \geq r\left|C_{H}+C_{V}\right|-\frac{1}{4}\left|C_{H}+C_{V}\right|\left(\frac{1}{2(4 r)^{-1}}\right)=\left|C_{V}+C_{H}\right| \frac{r}{2} .
\end{aligned}
$$

Lemma 2.2. For each given set of integers $\left\{m_{p}\right\}_{p \in \gamma}$ there exists for each $b \in D_{V}+$ $D_{H}$ a corresponding bond $f_{m}(b) \in \partial \gamma$ (the subindex $m$ indicates the dependence on $\left.\left\{m_{p}\right\}_{p \in \gamma}\right)$ with $f_{m}\left(b^{\prime}\right) \neq f_{m}(b)$ for $b^{\prime} \neq b, b^{\prime}, b \in D_{V}+D_{H}$, so that $j\left(a, \gamma, f_{m}(b)\right)$ is an odd number for all $b \in D_{V}+D_{H}$.

Proof. Assuming that for some $a$ and $i,\left(S_{a}, \gamma ; i\right) \neq \emptyset$, we have

$$
\sum_{b \in\left(S_{a}, \gamma ; i\right)}\left(S_{a} \mid b\right)\left(\partial_{\gamma}^{*} m\right)_{b}=0 .
$$


This follows from

$$
\begin{aligned}
& \sum_{b \in\left(S_{a}, \gamma ; i\right)} \sum_{p \ni b, p \in \gamma}(p \mid b)\left(S_{a} \mid b\right) m_{p} \\
& =\sum_{p \in \gamma, \partial p \cap\left(S_{a}, \gamma ; i\right) \neq \emptyset} m_{p} \sum_{b \in \partial_{p} \cap\left(S_{a}, \gamma ; i\right)}(p \mid b)\left(S_{a} \mid b\right)=0,
\end{aligned}
$$

since for any $p \in \gamma$,

$$
\sum_{b \in \partial p \cap\left(S_{a}, \gamma ; i\right)}(p \mid b)\left(S_{a} \mid b\right)=0
$$

Consider without loss of generality $b \in D_{V}$ and let $S_{a}$ be so that $b \in\left(S_{a}, \gamma ; i\right)$ for some $i$. (note that $\left(S_{a}, \gamma ; i\right) \cap A=b$ ). Then we have

$$
\sum_{b^{\prime} \in\left(S_{a}, \gamma ; i\right)}\left(\left(\partial_{\gamma}^{*} m\right)_{b^{\prime}}+k\left(A, b^{\prime}\right)\right)=k(A, b)= \pm 1
$$

So there is at least one odd number in

$$
\left\{\left(\partial_{\gamma}^{*} m\right)_{b^{\prime}}+k\left(A, b^{\prime}\right), b^{\prime} \in\left(S_{a}, \gamma ; i\right)\right\},
$$

and so, for each set of integers $\left\{m_{p}\right\}_{p \in \gamma}$ we may choose a bond $f_{m}(b) \in\left(S_{a}, \gamma ; i\right)$ satisfying desired condition. Injectivity is obvious.

Lemma 2.3. For $x \neq 0$ and $n$ odd

$$
\frac{C_{n}(x)}{C_{1}(x)} \leq 1
$$

Proof. First $C_{n}(x) / C_{1}(x)=I_{n}(x) / I_{1}(x)$. The modified Bessel functions satisfy the recursion relations:

$$
I_{n-1}(x)-I_{n+1}(x)=\frac{2 n}{x} I_{n}(x) \geq 0,
$$

which implies that $I_{n+1}(x) / I_{n-1}(x) \leq 1$ and the lemma follows directly.

Now we complete the majorization for $\left|\mu_{A}\right|^{1 /|\gamma|}$. We write, using $|A \cap \partial \gamma|=$ $\left|C_{H}+C_{V}\right|+\left|D_{H}+D_{V}\right|$

$$
\begin{aligned}
\mu_{A}(\gamma)= & C_{1}\left(\beta_{h}\right)^{-\left|C_{H}+C_{V}\right|} \sum_{\left\{m_{p}\right\}_{p \in \gamma}}\left\{\prod_{p \in \gamma} \frac{d_{m_{p}}\left(\beta_{g}\right)}{2 \pi}\right\} \\
& \times\left[\prod_{b^{\prime} \in D_{f_{m}}} \frac{C_{j\left(A, \gamma, b^{\prime}\right)}\left(\beta_{h}\right)}{C_{1}\left(\beta_{h}\right)}\right]\left\{\prod_{c \in \partial \gamma \backslash D_{f_{m}}} C_{j(A, \gamma, c)}\left(\beta_{h}\right)\right\},
\end{aligned}
$$

where $D_{f_{m}}:=\left\{f_{m}(b), b \in D_{V}+D_{H}\right\}$ with $f_{m}$ given as is Lemma $2.2\left(D_{f_{m}}\right.$ depends on $\left\{m_{p}\right\}_{p \in \gamma}$ ). Using $C_{n}(x) \leq 1$ and Lemma 2.3 we get for $\beta_{h} \neq 0$ :

$$
\left|\mu_{A}(\gamma)\right| \leq C_{1}\left(\beta_{h}\right)^{-\left|C_{H}+C_{V}\right|}\left\{\sum_{m \in \mathbb{Z}} \frac{\left|d_{m}\left(\beta_{\mathrm{g}}\right)\right|}{2 \pi}\right\}^{|\gamma|} .
$$


Choosing the constant $k$ in (6) so that $d_{0}\left(\beta_{g}\right) \geq 0$, a possible choice being $k=\beta_{\mathrm{g}}$ (for $m \neq 0, d_{m}\left(\beta_{\mathrm{g}}\right) \geq 0$ is automatically true), we get

$$
\begin{aligned}
\left|\mu_{A}(\gamma)\right| & \leq C_{1}\left(\beta_{h}\right)^{-\left|C_{H}+C_{V}\right|}\left|\varrho_{p}(0)\right|^{|\gamma|} \\
& \leq\left[C_{1}\left(\beta_{h}\right)^{-2 / r}\left(e^{2 \beta_{g}}-1\right)\right]^{|\gamma|},
\end{aligned}
$$

where the second inequality follows from Lemma 2.1. We conclude the existence of the bound

$$
\left\|\mu_{c}\right\| \leq C_{1}\left(\beta_{h}\right)^{-2 / r}\left(e^{2 \beta_{g}}-1\right) .
$$

According to the Appendix the convergence condition for the cluster expansion associated to our polymer expansion is, for fixed $r$ :

$$
C_{1}\left(\beta_{h}\right)^{-2 / r}\left(e^{2 \beta_{g}}-1\right) \leq \| \mu_{0} \mid .
$$

The corresponding regions are shown in Fig. 3 for two values of $r$.

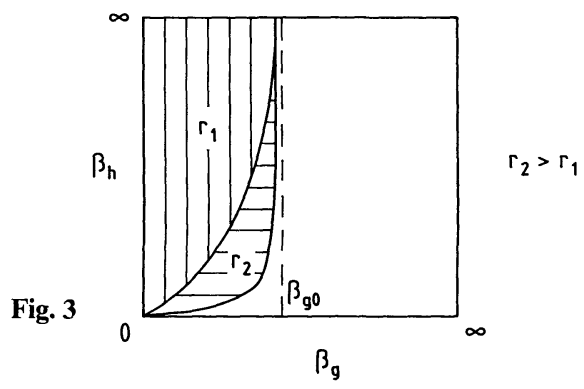
to

The convergence regions increase for increasing $r$ and converge asymptotically

$$
\left\{\left(\beta_{g}, \beta_{h}\right): \beta_{g}<\beta_{g_{0}}, \beta_{h} \neq 0\right\},
$$

where $\beta_{g_{0}}=1 / 2 \ln \left(1+\left\|\mu_{0}\right\|\right)$.

According to the results of [14], valid for systems like the one we are considering here, there is an analytical connection between the "confinement region" of the phase diagram (characterized by small values of $\beta_{g}$ ) and the "Higgs region" (characterized by large values of $\beta_{g}$ and $\beta_{h}$ ) for the expectation value of local observables. For this reason we should expect that the convergence of the cluster expansion extends to the Higgs region as well. Using the methods of [14], taking now $k=-\beta_{g}$ in (22) and using the simple bound $|A \cap \partial \gamma| /|\gamma| \leq 2$ (for $\gamma \neq \emptyset$ and $r>1$ ), one gets

$$
\left\|\mu_{c}\right\| \leq C_{1}\left(\beta_{h} / 2\right)^{-2} \varepsilon
$$

for any $\varepsilon>0$, provided $\beta_{h}>f_{\varepsilon}\left(\beta_{g}\right)$, where $f_{\varepsilon}\left(\beta_{g}\right): \mathbb{R}_{+} \rightarrow \mathbb{R}_{+}$is a monotonically increasing unbounded function of $\beta_{g}$, depending on $\varepsilon$. This shows that for each $\beta_{g}$ and for $\beta_{h}$ sufficiently large, condition (71) is satisfied and the convergence region for the cluster expansion may be extended from (57) to the full region $I_{c}$ of the $\left(\beta_{g}, \beta_{h}\right)$-phase diagram shown in Fig. 2: this holds in two or more space-time dimensions. 


\section{The Cluster Expansion}

At this point we return to expression (13) and (25), and writing

$$
\left\langle e^{i u_{N_{r}}}\right\rangle_{\Lambda}^{2}=\left\langle e^{i u_{N_{r}}}\right\rangle_{\Lambda}\left\langle e^{i u_{9 N_{r}}}\right\rangle_{\Lambda}
$$

(which holds if $\vartheta \Lambda=\Lambda$ ), we have at finite volume

$$
\varrho_{\text {voop }}^{(r)}=\frac{\left(\sum_{\Gamma \in G_{a d}^{A}} \prod_{\gamma \in \Gamma} \mu_{N_{r}}(\gamma)\right)\left(\sum_{\Gamma \in G_{a d}^{\Lambda}} \prod_{\gamma \in \Gamma} \mu_{\vartheta N_{r}}(\gamma)\right)}{\left(\sum_{\Gamma \in G_{a d}^{A}} \prod_{\gamma \in \Gamma} \mu_{M_{r}}(\gamma)\right)\left(\sum_{\Gamma \in G_{a d}^{A}} \prod_{\gamma \in \Gamma} \mu_{\emptyset}(\gamma)\right)},
$$

and taking the logarithm we write, according to the Appendix

$$
\ln \left(\left\langle e^{i u_{\vartheta N_{r}}}\right\rangle_{\Lambda}^{2} /\left\langle e^{i u_{M_{r}}}\right\rangle_{\Lambda}\right)=\sum_{\Gamma \in G^{A}} c_{\Gamma}\left(\mu_{N_{r}}^{\Gamma_{1}}+\mu^{\Gamma_{\vartheta N_{r}}}-\mu_{M_{r}}^{\Gamma}-\mu_{\emptyset}^{\Gamma_{\emptyset}}\right)
$$

(for the notation see the Appendix), where we assumed (56)-(58).

Relation (61) warrants the existence of the thermodynamical limit

$$
\ln \varrho_{\text {voop }}^{(r)}=\lim _{\Lambda \uparrow L} \ln \left\{\left\langle e^{i u_{N_{r}}}\right\rangle_{\Lambda}^{2} /\left\langle e^{i u_{M_{r}}}\right\rangle_{\Lambda}\right\}=\sum_{\Gamma \in G} c_{\Gamma}\left(\mu_{N_{r}}^{\Gamma_{1}}+\mu_{\vartheta N_{r}}^{\Gamma_{N}}-\mu_{M_{r}}^{\Gamma_{1}}-\mu_{\emptyset}^{\Gamma_{\emptyset}}\right),
$$

where $G=G^{L}$. Now we study the $\lim _{r \rightarrow \infty} \ln \varrho_{\text {voop. }}^{(r)}$. Following Fredenhagen and Marcu [1] one establish by simple geometrical reasoning that the right-hand side of (62) is equal to

$$
R_{r}:=\sum_{\Gamma \in J_{r}} c_{\Gamma}\left(\mu_{N_{r}}^{\Gamma_{1}}+\mu_{9 N_{r}}^{\Gamma_{9}}-\mu_{M_{r}}^{\Gamma}-\mu_{\emptyset}^{\Gamma}\right)
$$

where $J_{r}:=\left\{\Gamma \in G: \Gamma \nsim N_{r}\right.$ and $\left.\Gamma \in G: \Gamma \nsim \vartheta N_{r}\right\}$, where $\Gamma \nsim A$ means $\exists \gamma \in \Gamma: \partial \gamma \cap A \neq \emptyset$. One sees easily too that for any $s \in \mathbb{N}$,

$$
\left|R_{r}-R_{r+s}\right| \leq \sum_{\Gamma \in J_{r},\|\Gamma\| \geq r} 8\left|c_{\Gamma}\right| \max _{A \in\left\{N_{r}, M_{r}, \emptyset\right\}}\left|\mu_{A}\right|^{\Gamma} \leq r K\left(\frac{\left\|\mu_{c}\right\|}{\left\|\mu_{0}\right\|}\right)^{r},
$$

where the last inequality follows from (72), $K$ being a constant.

This last result implies that $\left\{R_{r}\right\}_{r \in \mathbb{N}}$ is a Cauchy sequence and that $\ln \varrho_{\text {voop }}^{(r)}$ converges and so

$$
\varrho_{\text {voop }}:=\lim _{r \rightarrow \infty} \varrho_{\text {voop }}^{(r)} \neq 0 .
$$

This holds in the convergence region $I_{c}$ of Fig. 2.

\section{Appendix}

In this appendix we fix some notations and remember some basic results on polymer and cluster expansions. For a review see [11, 10] or [1], appendix A.1. Our notation is essentially the same as [1].

For the two polymer expansions we treated in the last sections the compatibility relation between polymers is the following: two polymers $\gamma, \gamma^{\prime}$ are called compatible $\left(\gamma \sim \gamma^{\prime}\right)$ iff $\partial \gamma \cap \partial \gamma^{\prime}=\emptyset$ and incompatible $\left(\gamma \nsim \gamma^{\prime}\right)$ otherwise. We 
denote by $G_{c}^{\Lambda}$ the set of all polymers contained in $\Lambda \subset L$ and by $G_{a d}^{\Lambda}$ the set of all sets of compatible polymers (both $G_{c}^{\Lambda}$ and $G_{a d}^{\Lambda}$ contain the empty set).

A multi-index $\Gamma$ is a function $G_{c}^{\Lambda} \rightarrow \mathbb{N}$ and we denote by $G^{\Lambda}$ the set of all such functions. Two multi-indices $\Gamma, \Gamma^{\prime}$ are said to be incompatible $\left(\Gamma \nsim \Gamma^{\prime}\right)$ if there are $\gamma \in \Gamma, \gamma^{\prime} \in \Gamma^{\prime}$ with $\gamma \nsim \gamma^{\prime}$ and compatible $\Gamma \sim \Gamma$ otherwise $[\gamma \in \Gamma$ means $\Gamma(\gamma) \neq 0$.

For functions $f: G_{c}^{\Lambda} \rightarrow \mathbb{C}$ we use the multi-index notation

$$
f^{\Gamma}:=\prod_{\gamma \in \operatorname{supp} \Gamma} f(\gamma)^{\Gamma(\gamma)}
$$

for $\Gamma \in G^{\Lambda}$.

Given activities $\mu: G_{c}^{\Lambda} \rightarrow \mathbb{C}, \mu(\emptyset)=1$ we define the expectation value of a function $f$ by

$$
\langle f\rangle_{\mu, \Lambda}:=\frac{\sum_{\Gamma \in G_{a d}^{A}} \prod_{\gamma \in \Gamma} \mu(\gamma) f(\gamma)}{\sum_{\Gamma \in G_{a d}^{A}} \prod_{\gamma \in \Gamma} \mu(\gamma)},
$$

and one has in terms of formal power series the so-called cluster expansion for the logarithm of $\langle f\rangle_{\mu, \Lambda}$ :

$$
\log \langle f\rangle_{\mu, \Lambda}=\sum_{\Gamma \in G^{\Lambda}} c_{\Gamma}\left(f^{\Gamma}-1\right) \mu^{\Gamma},
$$

where $c_{\Gamma}$ are purely combinatorial coefficients given by (see [1])

$$
c_{\Gamma}:=\sum_{n=1}^{\infty} \frac{(-1)^{n}}{n} \mathscr{N}_{n}(\Gamma),
$$

where $\mathcal{N}_{n}(\Gamma)$ is the number of possibilities to write $\Gamma$ in the form $\Gamma=\Gamma_{1}+\cdots+\Gamma_{n}$, where $\Gamma_{i} \subset G_{c}^{\Lambda}, \Gamma_{i} \neq \emptyset$, (where we identify $\Gamma_{i} \subset G_{c}^{\Lambda}$ with its characteristic function). The function $c_{\Gamma}$ is called Ursell function and are also denoted in the literature (as in [10]) by the symbol $\Phi^{T}(\Gamma)$.

An important theorem says that if $\Gamma=\Gamma^{\prime}+\Gamma^{\prime \prime}$ with $\Gamma^{\prime} \sim \Gamma^{\prime \prime}\left(\Gamma^{\prime}, \Gamma^{\prime \prime} \neq 0\right)$ then $c_{\Gamma}=0$ (see $\left.[1,10,11]\right)$.

Beyond this the following result, which is fundamental for the study of the convergence of cluster expansions, has been established (see [1]):

Let $\|\left.\mu\left|:=\sup _{\gamma \neq \emptyset}\right| \mu(\gamma)\right|^{1 /|\gamma|}$, where $|\gamma|: G_{c}^{\Lambda} \rightarrow \mathbb{N},|\gamma|=0$ iff $\gamma=\emptyset$ is the size of $\gamma$ (in our case $|\gamma|$ is the number of plaquettes contained in $\gamma$ ) and define $\|\Gamma\|=\sum_{\gamma \in \operatorname{supp} \Gamma} \Gamma(\gamma)|\gamma|$, for $\Gamma \in G^{\Lambda}$. Then there exists a constant $K_{1}$ depending only on $\|\mu\|$ so that

$$
\sum_{\Gamma^{\prime} \nsim \Gamma}\left|c_{\Gamma^{\prime}}\right|\left|\mu^{\Gamma^{\prime}}\right| \leq K_{1}\|\Gamma\|
$$

provided

$$
\|\mu\| \leq\left\|\mu_{0}\right\|
$$

for a geometrically defined constant $\left\|\mu_{0}\right\|<1$. 
As a corollary one has (see [1]), under the same assumption, for any $n \in \mathbb{N}$,

$$
\sum_{\Gamma^{\prime} \nsim \Gamma,\left\|\Gamma^{\prime}\right\| \geq n}\left|c_{\Gamma^{\prime}}\right|\left|\mu^{\Gamma^{\prime}}\right| \leq\left(\frac{\|\mu\|}{\left\|\mu_{0}\right\|}\right)^{n}\|\Gamma\| K_{2},
$$

where $K_{2}$ is a constant.

Acknowledgement. I am indebted to K. Fredenhagen for discussions and for the suggestion to write this work, and to the referee for some comments.

\section{References}

1. Fredenhagen, K., Marcu, M.: Charged states in $\mathbb{Z}_{2}$ gauge theories. Commun. Math. Phys. 92, 81-119 (1983)

2. Fredenhagen, K.: Particle structure of gauge theories. Proceedings of the Summer School, "Fundamental Problems of Gauge Field Theory", Erice 1985

3. Fredenhagen, K., Marcu, M.: A confinement criterium for QCD with dynamical quarks. Phys. Rev. Lett. 56, 223 (1986)

4. Filk, T., Fredenhagen, K., Marcu, M., Slachanyi, K.: Charged states and order parameters in the Georgi-Glashow model. Desy Preprint 89-002 (1989)

5. Kondo, K.: Order parameter for charge confinement and phase structures in the lattice $U(1)$ Gauge-Higgs model. Prog. Theor. Phys. 74, 152-169 (1985)

6. Fradkin, E., Shenker, S.H.: Phase diagrams of lattice gauge theories with Higgs fields. Phys. Rev. D 19, 3682 (1979)

7. Borgs, C., Nill, F.: The phase structure of the abelian lattice Higgs model. A review of rigorous results. J. Stat. Phys. 47, 877 (1987)

8. King, C.: Deconfining phase transition in the $U(1)$ model with Wilson's action. Commun. Math. Phys. 105, 675-690 (1986)

9. Guth, A.: Existence proof of a non-confining phase in four dimensional $U(1)$ Lattice theory. Phys. Rev. D 21, 2291-2307 (1980)

10. Seiler, E.: Gauge theories as a problem of constructive quantum field theory and statistical mechanics. Lecture Notes in Physics, vol. 159. Berlin, Heidelberg, New York: Springer 1982

11. Brydges, D.C.: A short course on cluster expansions. Les Houches lecture notes (1984)

12. Slachanyi, K.: Non-local fields in the $Z(2)$ Higgs model. the global symmetry breaking and the confinement problem. Commun. Math. Phys. 108, 319-352 (1987)

13. Ginibre, J.: General formulation of Griffths' inequalities. Commun. Math. Phys. 16, 310 (1970)

14. Osterwalder, K., Seiler, E.: Gauge field theories on a lattice. Ann. Phys. 110, 440-471 (1978)

15. Barata, J.C.A., Wreszinski, W.F.: Absence of charged states in the $U(1)$ Higgs lattice Gauge theory. Commun. Math. Phys. 103, 637 (1986)

16. Jersák, J.: Lattice Higgs models in lattice Gauge theory - a chalenge in large-scale computing. Wuppertal, 1985

Communicated by L. Alvarez-Gaumé

Received July 1, 1989 
